\title{
Optimización de una cartera de inversión de renta variable
}

Armando Puebla Maldonado Universidad del Valle de México 


\title{
Optimización de una cartera de inversión de renta variable
}

\author{
Armando Puebla Maldonado iD \\ Universidad del Valle de México - México
}

\section{RESUMEN}

En este trabajo se hace una aplicación del modelo de selección de cartera de inversión propuesto por Harry Markowitz, para el caso de tres instrumentos financieros de renta variable que cotizan actualmente en el mercado de capitales de México. Para ello se tomó una muestra de 3 acciones de empresas mexicanas representativas que cotizan en el mercado accionario y que forman parte del Índice de Precios y Cotizaciones de la Bolsa Mexicana de Valores. Para la obtención de la cartera optima se generaron escenarios bajo optimización estática, dinámica y estocástica en Risk Simulator. Los resultados indican que cumpliéndose con las condiciones propuestas se obtienen los resultados esperados.

Para citaciones: Puebla Maldonado, A. (2021). Optimización de una cartera de inversión de renta variable. Panorama Económico, 29(3), 185-193.

Recibido: 13 de diciembre de 2020

Aprobado: 5 de abril de 2021

Autor de correspondencia: Armando Puebla Maldonado

armando.pueblama@uvmnet.edu

Editor: Andrés Escobar E. Universidad de Cartagena-Colombia.

Tipología IBN Publindex: Artículo Resultado de Investigación Científica y Tecnológica

Copyright: (C) 2021. Puebla Maldonado, A. Este es un artículo de acceso abierto, distribuido bajo los términos de la licencia https://creativecommons.org/licenses/bync-sa/4.0/ la cual permite el uso sin restricciones, distribución y reproducción en cualquier medio, siempre y cuando que el original, el autor y la fuente sean acreditados.

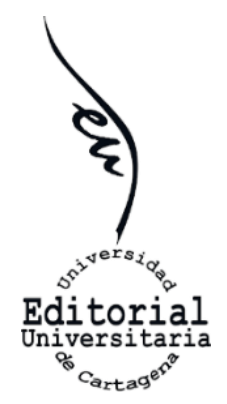

\section{Optimization of a variable income portfolio}

\section{ABSTRACT}

In this work, the investment portfolio selection model proposed by Harry Markowitz is applied for three financial instruments that are currently listed on the Mexican capital market. For this, a sample of 3 shares of representative Mexican companies that are listed in the stock market and that are part of the Mexican Stock Exchange Index was taken. To obtain the optimal portfolio, scenarios were generated under static, dynamic, and stochastic optimization in Risk Simulator. The results indicate that by fulfilling the proposed conditions the expected results are obtained.

Keywords: Portfolio; Risk; Performance; Linear programming; optimization. 


\section{INTRODUCCIÓN}

Para aplicar el modelo de portafolio en el ámbito financiero se propone como objetivo esencial construir una cartera de inversión eficiente a partir de la observación de un portafolio cuyos resultados inicialmente no maximizan el rendimiento de cada activo y que a su vez minimizan su riesgo y en consecuencia determinar la cartera optima aplicando Risk Simulator.

Ello implica responder a la pregunta de investigación; ¿es posible obtener portafolios óptimos cuando se presentan combinación de activos con tasas de rendimiento negativas? Desde que Harry Markowitz público su artículo en 1952 sobre teoría del portafolio destacan a nivel teórico tres importantes trabajos de investigación. Entre los desarrollos más importantes tenemos a Tobin que propone introducir en modelo el activo libre de riesgos (Tobin,1958). Posteriormente Sharpe (1963) desarrolla el Modelo de Evaluación de Activos para incluir los conceptos de riesgo sistemático y no sistemático. Finalmente, los trabajos de Fama y French $(1992,1993)$ sobre los modelos de arbitraje de tres factores ayudaron a comprender mejor las varianzas de las rentabilidades.

El documento se encuentra organizado de la siguiente manera. A continuación, se presenta el marco teórico, posteriormente se presenta la metodología, seguido de los resultados y discusión. Finalmente, se presentan las principales conclusiones de la investigación.

\section{MARCO TEÓRICO}

En el enfoque de carteras de Markowitz se planeta el caso de un inversionista que desea invertir de forma óptima cierta cantidad de dinero en el mercado de capitales. A dicha cantidad de capital se denota como C. Dado que el inversionista busca invertir el capital en activos a los que nos referiremos como activos disponibles que denotamos como $A_{i}$ $=\left(a_{1}, a_{2}, a_{3}, \ldots\right.$ an). La cuestión es, por tanto, decidir qué parte de su capital disponible invierte en cada uno de los activos disponibles $j, j=(1,2,3, \ldots n)$.

Una vez fijado los activos disponibles y el capital a invertir, se debe definir el porcentaje $O$, proporción a invertir en cada activo que se representa como $W=\left(W_{1}, W_{2}, W_{3}, \ldots W_{n}\right)$ y que se obtiene como $w_{j}=w_{i} / C$. Matemáticamente se expresa como el conjunto o cartera de valores de activos, como:

$\sum_{j=1}^{n} W i=100 \%$

Al asignar un porcentaje a cada activo disponible, también se requiere evaluar el rendimiento de cada uno de los activos. Se evalúa considerando el cambio de su cotización. De manera que la tasa de rendimiento $r_{t}$ de un activo en un intervalo de tiempo unidad que empieza en $t-1$ y acaba en $t$, es igual a la formula $r_{t}=\ln \left(p_{t} / p_{t-1}\right)$, siendo $\mathrm{pt}_{\mathrm{t}-1} \mathrm{y}$ pt las cotizaciones del activo en los tiempos $\mathrm{t}-1 \mathrm{y} \mathrm{t}$ respectivamente.

Por lo tanto, conociendo los activos disponibles $n=(1,2,3, \ldots n)$ el capital $C$, la tasa de rendimiento $r_{t}$ de una cartera de valores $j=(1,2,3, \ldots n)$ para un intervalo de tiempo 
unidad que empieza en t - 1 y acaba en t, el Rendimiento de un Portafolio (Rp) se calcula entonces como:

$R p=\sum_{j=1}^{n}$ Wiri

Para el cálculo de la cartera para tres activos, se tiene entonces:

$R p=w_{1} r_{1}+w_{2} r_{2}+w_{3} r_{3}$

Sujeto a las siguientes restricciones:

$\sum_{j=1}^{n=3}$ wiri $=100 \%$

Además:

$\sum_{j=1}^{n=3} w i=100 \%$

Donde: $0 \% \leq w_{i} \leq 100 \%$

Se asume también que el inversionista está interesado en realizar una inversión en un solo periodo, al que llamaremos tiempo de la inversión, compra los activos y construye la cartera de valores. A continuación, mantiene la cartera de valores inalterada durante un intervalo de tiempo prefijado (del orden de semanas, meses o años) hasta llegar al momento en el que espera obtener los beneficios debidos al cambio de la cotización de los activos, a dicho momento lo llamaremos función objetivo. En consecuencia, para modelar la tasa de rendimiento de cada activo disponible $j=(1,2,3, \ldots, n)$ como una variable aleatoria denotada por $r_{j}$ y suponiendo, además que su valor esperado $E\left(r_{i}\right)$ viene dada por la tasa esperada de rendimiento sobre la cartera como sigue para tres activos:

$R p=w_{1} E\left(r_{1}\right)+w_{2} E\left(r_{2}\right)+w_{3} E\left(r_{3}\right)$

Por su parte el riesgo individual se obtiene de la siguiente manera:

$\sigma=\sqrt{\frac{\sum_{i=1}^{n}\left(r_{t-\text { rpromedio })}\right.}{n}}$

Donde:

rt: riesgo individual de cada activo

rpromedio: riesgo promedio

Si se tiene un portafolio cuyo valor sólo depende de 3 factores de riesgo se tiene la siguiente matriz que se calcula de la siguiente manera:

$\begin{array}{lll}W_{1}^{2} \sigma_{11} & W_{1} W_{2} \sigma_{12} & W_{1} W_{3} \sigma_{13} \\ W_{2} W_{1} \sigma_{12} & W_{2}^{2} \sigma_{22} & W_{2} W_{3} \sigma_{23} \\ W_{3} W_{1} \sigma_{13} & W_{3} W_{2} \sigma_{23} & W_{3}^{2} \sigma_{33}\end{array}$


Donde:

$w_{i}=$ porcentaje invertido en cada una de las acciones, y $\sigma_{i k}$ es la covarianza de cada pareja de acciones.

En la diagonal principal de esta matriz aparecen las varianzas de las posibles combinaciones por pareja de los valores en la cartera, y fuera de la diagonal principal se encuentran sus covarianzas. Expresado como ecuación queda de la siguiente manera:

$\operatorname{Var}(R p)=w_{1}^{2} \sigma_{11}+w_{2}^{2} \sigma_{22}+w_{3}^{2} \sigma_{33}+2 w_{2} w_{1} \sigma_{12}+2 w_{3} w_{1} \sigma_{13}+2 w_{3} w_{2} \sigma_{32}$

De las ecuaciones y matrices anteriores se desprenden dos observaciones importantes. La primera es que la varianza de una cartera de activos riesgosos no es meramente las sumas de las varianzas respectivas, sino también está presente la covarianza entre los rendimientos de los activos; la segunda es que la varianza de una cartera de activos depende de los coeficientes de correlación, y es el valor de este coeficiente el que determina el conjunto de oportunidades de cartera de un inversionista.

Lo que se busca entonces es que los inversionistas puedan obtener tasas de rendimiento promedio esperadas más elevadas a un nivel de riesgo mínimo. Como normalmente esto es poco posible o probable que ocurra, dada la incertidumbre de los mercados financieros el inversionista se enfrenta al dilema entre rendimiento y riesgo, que se resuelve conociendo los porcentajes óptimos de inversión en cada activo a través de métodos cuantitativos de optimización.

\section{METODOLOGÍA}

En el ámbito empírico existen innumerables trabajos de investigación que buscan aplicar la teoría del portafolio en distintos escenarios y activos y con diferentes algoritmos matemáticos para obtener portafolios eficientes (Duvoba, 2005; Mas, 2011; Betancourt, 2013; León, 2015; Rivera, 1992; Munguía, 2013; Molina, 2015).

En lo que respecta a los trabajos de investigación usando programación lineal para optimizar carteras también encontramos que existe numerosas investigaciones. Por ejemplo, Prieto et al. (2014) utilizan planillas electrónicas para resolver el problema de optimización. En el mismo sentido, Zornoza, (2012 p. 57) afirma "El modelo...consiste en minimizar las desviaciones absolutas respecto a la rentabilidad media esperada de una cartera de valores bursátiles, utilizando la herramienta Solver que nos proporciona la hoja de cálculo". Finalmente, el trabajo de Cruz, Urrutia y Medina (2011) señalan que:

El objetivo de la investigación es desarrollar inicialmente una herramienta que arroje como resultado el portafolio de inversión óptimo con base en un conjunto de títulos del mercado de renta variable, para ello es necesario conocer el modelo matemático que permite llegar al resultado buscado.(p. 114)

Conociendo entonces que es posible obtener portafolios óptimos, el procedimiento que se siguió para la construcción del portafolio es el siguiente: 
Como primer paso, se obtuvo una muestra aleatoria simple elegida de 250 cotizaciones históricas de 3 empresas representativas del IPYC de la Bolsa Mexicana de Valores (BMV): Cemex, Femsa y Bimbo del año 2016. Posteriormente, se calculó la rentabilidad diaria de cada activo con base en los precios de cierre; en base a las siguiente formula: Rentabilidad relativa $=\mathrm{Ln}\left(\mathrm{P}_{t} / \mathrm{P}_{\mathrm{t}-1}\right)$. En seguida se obtuvieron los rendimientos esperados promedios para cada activo en base a la fórmula:

$r_{t+1=} \frac{\sum_{i=1}^{n} r_{i}}{n}$

Después se obtuvieron las volatilidades para cada activo:

$\sigma r=\sqrt{\frac{\sum_{i=1}^{n}\left(r_{\left.t-r_{t+1}\right)}\right.}{n}}$

Para conocer la dependencia lineal entre dos variables se obtuvieron las covarianzas:

$\operatorname{Cov}\left(r_{i}, r_{j}\right)=E\left[\left(r_{i}-E\left(r_{i}\right)\left(r_{j}-E\left(r_{j}\right)\right]\right.\right.$

En el caso de 3 activos:

$\operatorname{Cov}\left(r_{1}, r_{2}\right)=E\left[\left(r_{1}-E\left(r_{1}\right)\left(r_{2}-E\left(r_{2}\right)\right]\right.\right.$

$\operatorname{Cov}\left(r_{1}, r_{3}\right)=E\left[\left(r_{1}-E\left(r_{1}\right)\left(r_{3}-E\left(r_{3}\right)\right]\right.\right.$

$\operatorname{Cov}\left(r_{2}, r_{3}\right)=E\left[\left(r_{2}-E\left(r_{2}\right)\left(r_{3}-E\left(r_{3}\right)\right]\right.\right.$

Conociendo las covarianzas podemos obtener la matriz de ponderaciones suponiendo que el porcentaje invertido en cada activo es equiprobable, es decir que tengan el mismo porcentaje de inversión conforme a la siguiente tabla:

Tabla 1. Ponderaciones de varianza- covarianza

\begin{tabular}{lcl}
\hline$\left(\operatorname{Cov} r_{1} r_{1}\right)\left(W_{1}\right)\left(W_{1}\right)$ & $\left(\operatorname{Cov} r_{1} r_{2}\right)\left(W_{1}\right)\left(W_{2}\right)$ & $\left(\operatorname{Cov} r_{1} r_{3}\right)\left(W_{1}\right)\left(W_{3}\right)$ \\
\hline$\left(\operatorname{Cov} r_{1} r_{2}\right)\left(W_{1}\right)\left(W_{2}\right)$ & $\left(\operatorname{Cov} r_{2} r_{2}\right)\left(W_{2}\right)\left(W_{2}\right)$ & $\left(\operatorname{Cov} r_{2} r_{3}\right)\left(W_{2}\right)\left(W_{3}\right)$ \\
\hline$\left(\operatorname{Cov} r_{1} r_{3}\right)\left(W_{1}\right)\left(W_{3}\right)$ & $\left(\operatorname{Cov} r_{2} r_{3}\right)\left(W_{2}\right)\left(W_{3}\right)$ & $\left(\operatorname{Cov} r_{3} r_{3}\right)\left(W_{3}\right)\left(W_{3}\right)$ \\
& Fuente: Elaboración propia
\end{tabular}

\section{RESULTADOS}

Con la información de las cotizaciones diarias se obtuvo el rendimiento promedio y el riesgo de cada activo que se muestran en la tabla 2

Tabla 2. Rendimiento - Riesgo

\begin{tabular}{lcc}
\hline Acciones & Rendimiento promedio E(Ri) & Desviación o riesgo (бi) \\
\hline Cemex & $-0.04290 \%$ & $1.56173 \%$ \\
Bimbo & $-0.04031 \%$ & $1.33949 \%$ \\
Femsa & $0.03949 \%$ & $1.31614 \%$ \\
\hline
\end{tabular}

Fuente: Elaboración propia 
Como puede observarse se tienen 2 activos. Cemex y Bimbo que presentan tasas de rendimiento promedio negativo. En cuanto al riesgo, es ligeramente más alto para los activos que presentan rendimientos negativos. Esto puede deberse en parte a que en ese Bimbo presentaron perdidas en el mercado.

Para obtener la matriz de covarianza primero se obtuvo la matriz de correlación el cual arrojo los siguientes datos que se muestra en la tabla 3:

Tabla 3. Correlación

\begin{tabular}{llll}
\hline Acciones & Cemex & Bimbo & Femsa \\
\hline Cemex & 1 & 0.246748295 & 0.055893328 \\
Bimbo & 0.246748295 & 1 & 0.060914494 \\
Femsa & 0.055893328 & 0.060914494 & 1 \\
\hline
\end{tabular}

Fuente: Elaboración propia

Se puede apreciar que la correlación más alta se presenta entre Cemex y Bimbo, aunque el valor de 0.24 es bajo. Una vez obtenido la matriz de correlación el siguiente paso es conocer la covarianza la cual representa una medida de la tendencia de los rendimientos a moverse en la misma dirección cuyos resultados se resumen en la siguiente tabla:

Tabla 4: Varianza - Covarianza

\begin{tabular}{llll}
\hline Acciones & Cemex & Bimbo & Femsa \\
\hline Cemex & 0.00024390 & 0.0000516 & 0.00001149 \\
Bimbo & 0.00005162 & 0.0001794 & 0.00001074 \\
Femsa & 0.00001149 & 0.0000107 & 0.00017322 \\
\hline
\end{tabular}

Fuente: Elaboración propia

A partir de la matriz de varianza covarianza se obtuvo la matriz de ponderaciones, el cual como vimos suponiendo que se invierten las mismas proporciones en cada una de las acciones queda como se muestra en la tabla 5.

Tabla 5: Ponderaciones

\begin{tabular}{llllc}
\hline & & & & \\
& & Cemex & Bimbo & Femsa \\
\hline Peso & & $33.33 \%$ & $33.33 \%$ & $33.33 \%$ \\
$33.33 \%$ & Cemex & 0.000029 & 0.0000057 & 0.00000128 \\
$33.33 \%$ & Bimbo & 0.0000053 & 0.0000199 & 0.00000119 \\
$33.33 \%$ & Femsa & 0.0000018 & 0.0000012 & 0.00001924 \\
& Suma & 0.0003410 & 0.00002686 & 0.00002171 \\
\hline
\end{tabular}

Fuente: Elaboración propia

Con los datos anteriores se obtuvo el rendimiento del portafolio $(R p)=2.2844 \%$ y el riesgo del portafolio $\operatorname{Var}(\mathrm{Rp})=0.81 \%$ asignando un $5 \%$ a Cemex y a Bimbo y a Femsa del $90 \%$ en función rendimiento y riesgo individual de los activos como se muestra en la tabla 6. Para la obtención de los beneficios o rendimientos anualizados se utilizó la fórmula de la Media Geométrica1.

${ }^{1} M G=\sqrt{\sum_{i=1}^{n}\left(1+r_{i}\right)^{\wedge}} n$ 
Tabla 6. Portafolio de inversión

\begin{tabular}{|c|c|c|c|c|c|c|c|c|}
\hline $\begin{array}{l}\text { Descripc } \\
\text { ión del } \\
\text { Tipo de } \\
\text { Activo }\end{array}$ & $\begin{array}{l}\text { Benefici } \\
\text { os } \\
\text { Anualiza } \\
\text { dos }\end{array}$ & $\begin{array}{l}\text { Riesgo } \\
\text { de } \\
\text { Volatilid } \\
\text { ad }\end{array}$ & $\begin{array}{l}\text { Asignaci } \\
\text { ón de } \\
\text { Pesos }\end{array}$ & $\begin{array}{l}\text { Asignaci } \\
\text { ón } \\
\text { Mínima } \\
\text { Requeri } \\
\text { da } \\
\end{array}$ & $\begin{array}{l}\text { Asignaci } \\
\text { ón } \\
\text { Máxima } \\
\text { Requerid } \\
\text { a }\end{array}$ & $\begin{array}{l}\text { Benefic } \\
\text { io para } \\
\text { el Ratio } \\
\text { de } \\
\text { Riesgo } \\
\end{array}$ & $\begin{array}{l}\text { Clasificaci } \\
\text { ón de } \\
\text { Beneficios } \\
\text { (Alto- } \\
\text { Bajo) } \\
\end{array}$ & $\begin{array}{l}\text { Clasifica } \\
\text { ción de } \\
\text { Riesgo } \\
\text { (Bajo- } \\
\text { Alto) } \\
\end{array}$ \\
\hline Cemex & $2.2843 \%$ & $1.561 \%$ & $33.33 \%$ & $5.00 \%$ & $35.00 \%$ & 1.4631 & 3 & 3 \\
\hline Bimbo & $2.2843 \%$ & $1.339 \%$ & $33.33 \%$ & $5.00 \%$ & $35.00 \%$ & 1.7055 & 2 & 2 \\
\hline Femsa & $2.2847 \%$ & $1.316 \%$ & $33.33 \%$ & $5.00 \%$ & $35.00 \%$ & 1.7360 & 1 & 1 \\
\hline $\begin{array}{l}\text { Total, de } \\
\text { Cartera }\end{array}$ & $2.2844 \%$ & $0.81 \%$ & $100.00 \%$ & & & & & \\
\hline $\begin{array}{l}\text { Relación } \\
\text { Retorno } \\
\text { / Riesgo }\end{array}$ & 2.8063 & & & & & & & \\
\hline
\end{tabular}

Fuente: Elaboración propia

El beneficio para la tasa de riesgo se obtiene dividiendo los beneficios entre el riesgo de cada activo. En la clasificación de beneficio y de riesgo se jerarquizó en función de los valores más altos a más bajos. Sin embargo, ¿es posible mejorar el rendimiento del portafolio que a su vez disminuya el riesgo aplicando una metodología de optimización matemática? Para ello, se procede a realizar 3 tipos de simulaciones de optimización en el programa de Risk Simulator:

a. Optimización estática: Mediante un modelo discreto o estático con el fin de obtener una cartera optima en base a las ponderaciones.

b. Optimización dinámica: Mediante un modelo dinámico se ejecuta la simulación para 1000 iteraciones a los valores asignados.

c. Optimización estocástica: Similar a la optimización dinámica con la característica de que el proceso se repite varias veces y cada variable de decisión final tendrá su propia tabla de pronóstico donde se indica su rango óptimo.

En los tres tipos de simulaciones, los resultados muestran los mismos valores (Tabla 7). Para Bimbo y Femsa, el portafolio indica que habría que invertirle el 35\% y en el caso de Cemex del 30\%. Lo mismo sucede con el rendimiento y riesgo de la cartera que se mantuvo en $2.2845 \%$ y $0.81 \%$. Se observa que mejora la relación retorno/Riesgo, pues paso de 2.8306 a 2.8063. Esto es importante ya que entre más alto mayor es el beneficio que se obtiene de la inversión.

Tabla 7: Resultado de las simulaciones

\begin{tabular}{|c|c|c|c|c|c|c|c|c|}
\hline $\begin{array}{l}\text { Descripc } \\
\text { ión del } \\
\text { Tipo de } \\
\text { Activo }\end{array}$ & $\begin{array}{l}\text { Beneficio } \\
\text { s } \\
\text { Anualiza } \\
\text { dos }\end{array}$ & $\begin{array}{l}\text { Riesgo } \\
\text { de } \\
\text { Volatili } \\
\text { dad }\end{array}$ & $\begin{array}{l}\text { Asigna } \\
\text { ción de } \\
\text { Pesos }\end{array}$ & $\begin{array}{l}\text { Asignac } \\
\text { ión } \\
\text { Mínima } \\
\text { Requeri } \\
\text { da }\end{array}$ & $\begin{array}{l}\text { Asignac } \\
\text { ión } \\
\text { Máxima } \\
\text { Requeri } \\
\text { da }\end{array}$ & $\begin{array}{l}\text { Beneficio } \\
\text { para el } \\
\text { Ratio de } \\
\text { Riesgo }\end{array}$ & $\begin{array}{l}\text { Clasificac } \\
\text { ión de } \\
\text { Beneficio } \\
\text { s (Alto- } \\
\text { Bajo) }\end{array}$ & $\begin{array}{l}\text { Clasificac } \\
\text { ión de } \\
\text { Riesgo } \\
\text { (Bajo- } \\
\text { Alto) }\end{array}$ \\
\hline Cemex & $2.2843 \%$ & $1.561 \%$ & $30 \%$ & $5.00 \%$ & $35.00 \%$ & 1.4631 & 3 & 3 \\
\hline Bimbo & $2.2843 \%$ & $1.339 \%$ & $35 \%$ & $5.00 \%$ & $35.00 \%$ & 1.7055 & 2 & 2 \\
\hline Femsa & $2.2847 \%$ & $1.316 \%$ & $35 \%$ & $5.00 \%$ & $35.00 \%$ & 1.7360 & 1 & 1 \\
\hline $\begin{array}{l}\text { Total, de } \\
\text { Cartera }\end{array}$ & $2.2845 \%$ & $0.81 \%$ & $\begin{array}{c}100.00 \\
\%\end{array}$ & & & & & \\
\hline $\begin{array}{l}\text { Relación } \\
\text { Retorno } \\
\text { / Riesgo } \\
\end{array}$ & 2.8306 & & & & & & & \\
\hline
\end{tabular}

Fuente: Elaboración propia en base a los resultados generados por Risk Simulator 
Cabe también destacar que los valores obtenidos por la simulación estocástica o la optimización dinámica se logró el objetivo inicial de mejorar el rendimiento que se observa en la tabla 8. Razón por la cual, el riesgo prácticamente en los tres escenarios se mantuvo constante.

\begin{tabular}{llll}
\hline \multicolumn{4}{c}{ Tabla 8. Resultados } \\
\hline \hline Fecha de Generación del Reporte: & Tuesday, January 26, 2021 7:50:00 PM & \\
Tiempo Total de la Ejecución: & 70.55 secs. & \\
Nombre del Perfil: & Optimización & \\
Tipo de Optimización: & Stochastic Optimization & \\
Valor Inicial de los Objetivos: & 2.806344 & & \\
Valor Optimizado de los Objetivos: & 2.830615 & & Valor Final \\
Restricciones: & & Valor Inicial & 0.29 \\
$\quad$ Requerimiento & & 0.333333 & 0.35 \\
$\quad$ Cemex & & 0.333333 & 0.35 \\
\hline Bimbo & & 0.333333 & 0.35
\end{tabular}

Fuente: Elaboración propia a partir de Risk Simulator

\section{DISCUSIÓN Y CONCLUSIONES}

De los resultados obtenidos se destaca la importancia de aplicar la aplicación de un programa para optimizar portafolios de inversión. Por supuesto, los resultados tienden a variar en la medida en que las cotizaciones también lo hacen por lo que no siempre garantizan ganancias continuas a largo plazo, aun y cuando la metodología utilizada buscar mejorar el rendimiento y minimizar el riesgo.

Aunque el modelo de optimización utilizado mejora los resultados obtenidos inicialmente, no significa que mejoren aplicando otras metodologías utilizadas en la literatura como el procedimiento de los multiplicadores de Lagrange, el criterio de media-varianza, el procedimiento de Desviación Media Absoluta, la metodología de Fuzzy o de programación lineal.

Finalmente, la utilización de diferentes herramientas para optimizar portafolios de inversión es fundamental para obtener los resultados esperados. En este documento, se utilizó el software Risk Simulator como una de las herramientas de optimización. Sin embargo, cabe señalar que existen otros enfoques como la evaluación de activos, el cálculo de la frontera eficiente, los modelos de arbitraje, los cuales son ampliamente utilizados por los analistas financieros para tomar las mejores decisiones de inversión.

\section{REFERENCIAS}

Betancourt, K. (2013). Teoría de Markowitz con metodología EWMA para la toma de decisión sobre cómo invertir su dinero. Atlantic Review of Economics, (1st Volume), "pp.32-43"

Cruz Trejos, E., \& Urrutia Mosquera, J., \& Medina Varela, P. (2011). Un modelo dual para portafolios de inversión. Scientia Et Technica, Xvii (47), 113-118. 
Dubova, Irina, La validación y aplicabilidad de la teoría de portafolio en el caso colombiano. Cuadernos de Administración [en línea] 2005, 18 (julio-diciembre): [Fecha de consulta: 24 de julio de 2018] Disponible en: http://www.redalyc.org/articulo.oa?id=20503010 ISSN 0120-3592

Fama, E. F.; French, K. R. (1993). "Common risk factors in the returns on stocks and bonds". Journal of Financial Economics 33: 3.

Fama, E. F.; French, K. R. (1992). "The Cross-Section of Expected Stock Returns". The Journal of Finance 47 (2): 427.

Konno, H., \& Yamazaki, H. (1991). Mean-Absolute Deviation Portfolio Optimization Model and Its Applications to Tokyo Stock Market. Management Science, 37(5), 519-531. Consultado de http://www.jstor.org/stable/2632458

León, E. B. (2015). Valoración de Carteras de Acciones en el Mercado Continuo (Master's thesis). [Fecha de consulta: 13 de junio de 2018] Disponible en: http://diposit.ub.edu/dspace/bitstream/2445/66816/1/TFG-ADE-Benages-Enrique-juliol15.pdf

Markowitz, H. (1952). Portfolio Selection. The Journal of Finance, 7(1), 77-91. DOI:10.2307/2975974

Mas, M. M. (n.d.). Una manera de optimizar un portfolio financiero. In file:///C:/Users/38550/Downloads/1159-Texto\%20del\%20art\%C3\%ADculo-3027-1-10-

20131227.pdf Consultado en Junio 18, 2018, de file://C:/Users/38550/Downloads/1159Texto\%20del\%20art\%C3\%ADculo-3027-1-10-20131227.pdf

Molina, J. S. (2015). Solución del problema de la selección de un portafolio mediante programación semi-infinita (Master's thesis).

Mungia, F. G. (2013). Métodos alternativos para la selección de portafolios de inversión en Mercados Emergentes (Master's thesis). Consultado en Junio 18, 2018, from file:///C:/Users/38550/Downloads/1159-Texto\%20del\%20art\%C3\%ADculo-3027-1-1020131227.pdf

Prieto U, J., González C, M., \& Arce E, A. (2014). Aplicación de Programación Lineal en la Gestión de Carteras.. FPUNE Scientific, 4(4). Recuperado de http://www.une.edu.py:82/fpune_scientific/index.php/fpunescientific/article/view/82

Rivera, C. F. (1992). Un Modelo de Optimización para Carteras de Inversión (Master's thesis).

Sharpe, William F. (1963). "Un modelo simplificado para el análisis de la cartera". Ciencia Gerencial. 9 (2): 277-93. DOI: 10.1287 / mnsc.9.2.277.

Sharpe, William F. (1964). "Precios de capital de los activos: una teoría del equilibrio del mercado en condiciones de riesgo". Revista de Finanzas. XIX (3): 425-442. DOI: 10.2307 / 2977928. JSTOR 2977928.

Richard Nelson and Bela Balassa, eds., Economic Progress: Private Values and Public Policy (Essays in Honor of William Fellner), Amsterdam: North-Holland, 235-62.

Tobin, James and William C. Brainard (1977). "Asset Markets and the Cost of Capital". In Tobin, James (1958). «Liquidity Preference as Behavior Towards Risk». Review of Economic Studies 25.1: 65-86.

Zornoza, J. F. (2012, April). Modelo de selección de cartera con Solver. Modelling in Science Education and Learning, 5, 57-63. DOl: 10.4995/msel.2012.2133 